\title{
Microstructural Characterization of Bio Based Hydroxyapatite Infused Polycaprolactone Forcespun Fibers
}

\author{
Deepa Kodali and Vijay Rangari \\ Tuskegee University, Tuskegee, Alabama, United States
}

Hydroxyapatite (HA) belongs to a class of calcium phosphate materials and has similar physical and chemical characteristics as that of the natural bone. Owing to its biocompatible, osteoconductive, and bioactive nature, $\mathrm{HA}\left[\mathrm{Ca}_{10}\left(\mathrm{PO}_{4}\right)_{6}(\mathrm{OH})_{2}\right]$ is extensively used in biomedical applications, such as bone regeneration, dental implants and drug delivery systems [1]. Recently development of HA based fibrous scaffolds have become the focus of numerous studies due to its hemostatic and bone healing properties. Various techniques like sol-gel process, solid state reaction, hydrothermal reaction, co-precipitation reaction were available for the production of HA. However, obtaining the nanoscale HA through synthetic methods were rather complicated, expensive, hazardous and time consuming. On the contrary, the natural HA that can be produced from biowaste such as fish bone, coral, sea shells, egg shells, chicken bone etc., are rich in calcium and serves as promising approach to annihilate the environmental related problems [2]. Due to the ultrafine structure and significant functional properties of nanoscale HA, it has been widely used in the preparation of polymer composites for biomedical applications [3]. The exceptional mechanical properties combined with the biodegradability and biocompatability made Polycaprolactone (PCL) an undeniable source of application in regenerative medicine and constituent materials in nanocomposites. Recent studies emphasized that the blending of PCL and HA can enhance the cell proliferation and differentiation [4]. The objective of the present study is to analyze the microstructure and chemical composition of the forcespun PCL fibers infused with HA derived from fish scales using Field Emission Scanning Electron Microscopy (FE-SEM) and Energy Dispersive Spectroscopy (EDS).

The sun dried fish scales of Carpa fish were purchased from Nizona Corporation, India. The powdered PCL with a molecular weight of 50000 was purchased form Polysciences, Inc (Warrington, PA, USA). The solvent chloroform with ACS reagent $\geq 99.8 \%$ was purchased from Sigma-Aldrich (St. Louis, MO, USA).

In the present study, the HA was synthesized from the dried fish scales by calcination at $800^{\circ} \mathrm{C}$ for three hours using a high temperature pressure reactor. Thus obtained powder was further ball milled for $2 \mathrm{~h}$ at 2000rpm using MTI compact nano agitator bead mill. This powder was further washed with ethanol and dried for $24 \mathrm{~h}$ at room temperature. The polymer solution was prepared by dissolving the $16 \mathrm{wt} \%$ of powdered PCL in chloroform. 1wt\% of the nanoscale HA was added to the polymer solution and is mixed with the magnetic stirrer at 170rpm for $3 \mathrm{~h}$. The PCL/HA fibers were obtained by forcespinn technique where the solution mixture was rotated at $5000 \mathrm{rpm}$ speed using a Cyclone L-1000M apparatus from Fiberio. The as-prepared fibers were collected and stored under desiccation. The samples were analyzed using Field Emission Scanning Electron Microscope (JOEL JSM-7200F) which was equipped with Energy Dispersive X-Ray Analyzer, EX-37001. The samples were sputter coated with gold-palladium for $5 \mathrm{~min}$ at $5 \mathrm{~V}$ and $10 \mathrm{~mA}$ prior to testing in Hummer 6.2 sputter coater purged with argon gas.

The SEM micrographs of PCL neat and 1wt\% PCL/HA fibers are shown in figure 1(a). The average diameter of PCL neat fibers is observed to be $1.19 \mu \mathrm{m}$ whereas PCL/HA fibers showed $1.28 \mu \mathrm{m}$. The addition of the nanoparticles has increased the diameter of the fibers. Furthermore, the PCL/HA fibers showed small beads. The EDS spectra shown in figures 1(b) and 1(c) confirms the elemental composition 
of HA. The EDX mapping in figure 2 shows the distribution of carbon, oxygen, calcium and phosphorous. The elemental distribution of $\mathrm{Ca}$ and $\mathrm{P}$ suggests that the nano HA particles are well dispersed in polymer solution and can be effectively used to produce composite fibers.

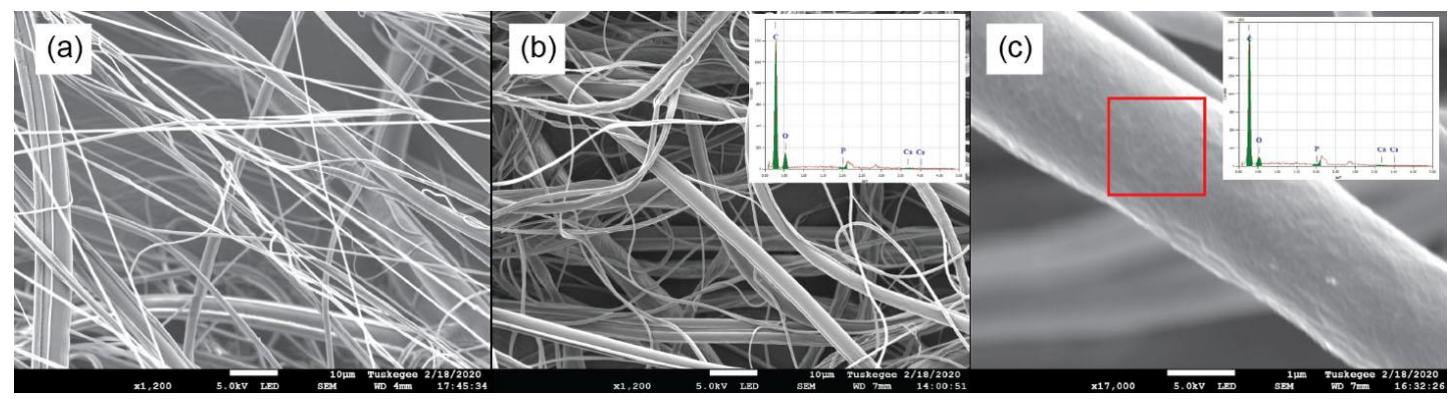

Figure 1. SEM micrographs of forcespun PCL/HA fibers with EDS spectra showing chemical composition (a) PCL (b) PCL with 1wt\% of HA (c) Expanded view of PCL/HA fiber.

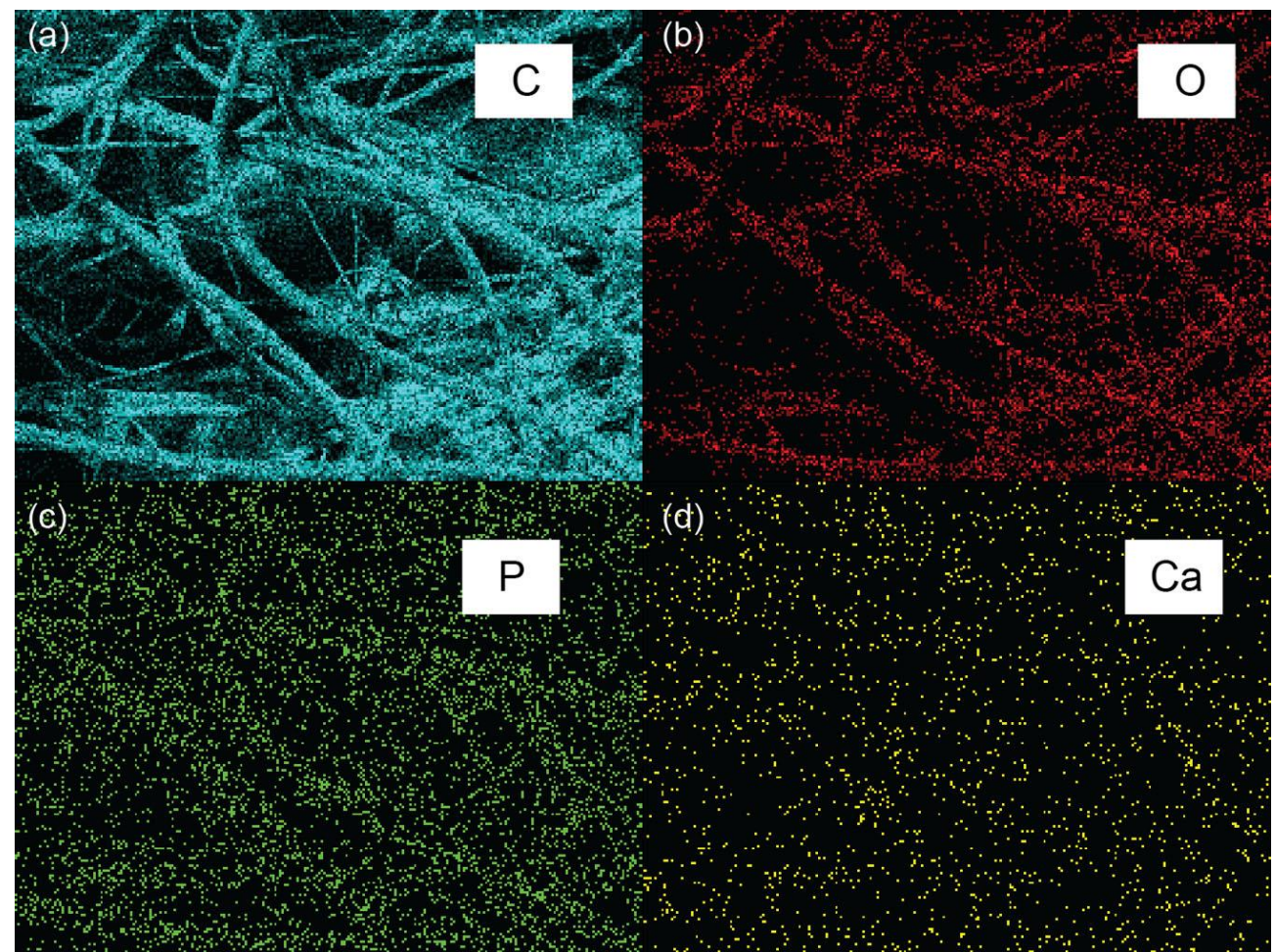

Figure 2. EDX mapping of forcespun PCL fibers infused with $1 \mathrm{wt} \%$ of HA showing distribution of (a) Carbon (b) Oxygen (c) Phosphorous and (d) Calcium.

\section{References}

[1]. Lee, J. H.; Kim, Y. J. Ceram. Int. 40 (2014), p, 3361-69.

[2]. Pokhrel, S. Adv. Chem. Eng. Sci. 08 (2018), p, 225-40.

[3]. Rahman, M. M.; Netravali, A. N.; Tiimob, B. J.; Apalangya, V.; Rangari, V. K. J. Appl. Polym. Sci. 133 (2016), 43477.

[4]. Chen, M.; Parsons, A. J.; Felfel, R. M.; Rudd, C. D.; Irvine, D. J.; Ahmed, I. J. Mech. Behav. Biomed. Mater. 59 (2016), p, 78-89. 